\title{
Revisión de la vía clínica "inicio programado en hemodiálisis"
}

\author{
Moisés Navarro Bermúdez
}

Enfermero y licenciado antropología social y cultural, Servicio de Nefrología. Hospital de Jerez. Cádiz

\section{Sr. Director:}

Una vía clínica pretende ser un instrumento institucionalizado, donde todos los profesionales del servicio sepan en todo momento todas las actividades y acciones que tienen que llevar a cabo el tiempo que dura esta actividad asistencial y los usuarios/familiares conozcan desde el primer momento toda la información de la asistencia que se les va a prestar.

La vía clínica de inicio programado a hemodiálisis, se implementó en marzo 2010 en la unidad de hemodiálisis del Hospital de Jerez, y después de un año de la misma y con los primeros 15 casos ya atendidos, hemos pretendido hacer una reflexión sobre la misma, al objeto de establecer mejoras.

Esta vía clínica está compuesta por los siguientes elementos ${ }^{1,2}$ :

- Una matriz temporal, donde se detallan por día las actividades de todos los profesionales implicados en la vía clínica, y se especifican otros aspectos importantes como son las pruebas complementarias, dieta y medicación $n^{3,4,5,6,7,8,9}$.

Correspondencia:

Moisés Navarro Bermúdez

c/ Rodrigo de Bastidas $12,2^{\circ} \mathrm{A}$

11500 Pto Sta Maria (Cádiz)

e-mail:monavber@ono.com
- Hoja de variaciones. Donde se detallan tanto las desviaciones de la vía como las acciones realizadas o su justificación.

- Hoja de información al paciente y/o familiar. Incluye una serie de ilustraciones que informan al paciente sobre la evolución más frecuente del proceso. Es una versión gráfica simplificada de la matriz para una fácil comprensión del paciente.

- Encuesta de satisfacción del paciente y/o familiar. Se entrega al paciente para que exprese de forma anónima su opinión sobre la atención y cuidados prestados. Engloba preguntas respecto a la información recibida, la atención, aspectos de hostelería, etcétera.

Para ello, llevamos a cabo una sesión clínica acreditada para analizar la vía clínica, y establecer medidas de consenso que mejorasen, si fuera necesario la misma.

\section{Metodología}

Convocamos una sesión clínica acreditada, dirigida a todo el personal de la unidad, con el siguiente objetivo:

- Analizar y conocer los primeros casos que han sido incluidos en la vía clínica, al objeto de mejorar la calidad de los cuidados prestados a los pacientes nefrológicos incluidos en dicha vía. 
La sesión clínica se celebró en nuestra unidad, fue acreditada con 2,35 créditos y asistieron un total de 20 profesionales (15 enfermeros, 3 nefrólogos y 2 auxiliares de enfermería).

Esta sesión clínica pretendía desarrollar las siguientes competencias:

\section{Los médicos nefrólogos}

a. Orientación al ciudadano: respeto por sus derechos y concretamente la siguientes

b. Trabajo en equipo

c. Actitud de progreso y desarrollo profesional

d. Orientación a resultados

\section{Enfermeros de hemodiálisis}

a. Orientación al ciudadano

b. Atención Sanitaria Integral

c. Personalización de los cuidados

d. Trabajo en equipo

e. Actitud de aprendizaje y mejora continua

f. La eficiencia. Uso adecuado de los recursos

g. Orientación de resultados

\section{Datos recogidos de los pacientes atendidos:}

De los 15 pacientes que recogimos la información, sólo 4 pacientes estuvieron 3 sesiones de hemodiálisis, que era el número de sesiones máximas que teníamos preestablecido en la matriz de la vía, 5 pacientes tuvieron 4 sesiones, 4 pacientes 5 sesiones y el resto estuvieron más cinco de sesiones.

Sólo uno de los pacientes lo sacamos de la vía clínica por recuperación de la función renal.

\section{Las principales variaciones que nos encontramos en la vía fueron:}

- Problemas en la canalización de la fístula arteriovenosa, que precisó que su diálisis se desarrollara en unipunción.
- Pacientes con anemia, que precisaron tratamientos con eritropoyetina, e incluso dos de ellos necesitaron de transfusiones sanguíneas.

- Pacientes con problemas de hipotensiones durante la sesión de hemodiálisis, que precisaron la reposición de líquidos durante la misma.

- Como otros, encontramos retrasos en el alta del paciente, por problemas con los centros periféricos a la hora de buscarles sitios en dicho centro.

\section{Con todos estos datos obtenidos, sacamos las siguientes conclusiones:}

- Aumentar las sesiones de hemodiálisis de la vía clínica, de tres a cinco sesiones.

- Incluir dentro de la medicación a administrar dentro las sesiones de hemodiálisis, la administración de eritropoyetina post-hemodiálisis y venofer IV.

- Mejorar el circuito de comunicación con centros periféricos, para agilizar los traslados a los mismos.

- Evitar punciones innecesarias, cuando el acceso vascular sea dificultoso, considerando la unipunción como aceptable.

Por tanto la matriz de la vía clínica de inicio programado en hemodiálisis y la hoja informativa al paciente quedaría de la siguiente manera. 
[ M. Navarro Bermúdez ]

Revisión de la vía clínica "inicio programado en hemodiálisis"

\begin{tabular}{|c|c|c|c|c|c|c|}
\hline & DÍA 0 & $\begin{array}{l}\text { DÍA } 1 \\
1^{\text {a }} \text { diálisis }\end{array}$ & $\begin{array}{l}\text { DÍA } 2 \\
2^{\mathrm{a}} \text { diálisis }\end{array}$ & $\begin{array}{l}\text { DÍA } 3 \\
3^{\text {a }} \text { diálisis }\end{array}$ & $\begin{array}{l}\text { DÍA } 4 \\
4^{\text {a }} \text { diálisis }\end{array}$ & $\begin{array}{l}\text { DÍA } 5 \\
5^{\text {a }} \text { diálisis }\end{array}$ \\
\hline $\begin{array}{l}\text { CIRCUITO DE } \\
\text { CONTACTO }\end{array}$ & $\begin{array}{l}\text { El nefrólogo con- } \\
\text { tacta con la unidad } \\
\text { de agudos (Ext } \\
\text { 099) para progra- } \\
\text { mación de paciente }\end{array}$ & & & & & \\
\hline $\begin{array}{l}\text { ACTUACIÓN } \\
\text { MÉDICA }\end{array}$ & $\begin{array}{l}\text { Confirmación de ci- } \\
\text { ta. Entrega de hoja } \\
\text { de información al } \\
\text { paciente }\end{array}$ & $\begin{array}{l}\text { HC } \\
\text { Exploración física } \\
\text { Petición de pruebas } \\
\text { Revisión del tratamiento }\end{array}$ & $\begin{array}{l}\text { Se evaluará al } \\
\text { paciente sólo si se } \\
\text { producen } \\
\text { incidencias }\end{array}$ & $\begin{array}{l}\text { Se evaluará al } \\
\text { paciente sólo si se } \\
\text { producen } \\
\text { incidencias }\end{array}$ & $\begin{array}{l}\text { Se evaluará al } \\
\text { paciente sólo si } \\
\text { se producen } \\
\text { incidencias }\end{array}$ & $\begin{array}{l}\text { HC } \\
\text { Exploración física } \\
\text { Elaboración de } \\
\text { informe de alta. }\end{array}$ \\
\hline $\begin{array}{l}\text { CUIDADOS } \\
\text { DE } \\
\text { ENFERMERÍA }\end{array}$ & $\begin{array}{l}\text { El enfermero le } \\
\text { entregará la hoja } \\
\text { de información al } \\
\text { paciente/familia }\end{array}$ & $\begin{array}{l}\text { Protocolo de acogida del } \\
\text { paciente en HD: } \\
\text { 1. Breve descripción de la } \\
\text { diálisis } \\
\text { 2. Se le mostrará la unidad } \\
\text { 3. Conceptos básicos de } \\
\text { FAV } \\
\text { 4. Conceptos básicos sobre } \\
\text { peso, dieta y restricción de } \\
\text { líquidos } \\
\text { Se le facilitará una "Guía } \\
\text { para pacientes con trata- } \\
\text { miento de HD" } \\
\text { Se le realizará valoración } \\
\text { enfermera, escalas clinimé- } \\
\text { tricas, se abrirá plan de cui- } \\
\text { dados e historia de diálisis. } \\
\text { Biompedancia pre-post } \\
\text { diálisis } \\
\text { Duración de tratamiento } \\
\text { (1ª sesión): } \\
2 \text { h de HD. Sin pérdidas. } \\
\text { Perfusión continua de Ma- } \\
\text { nitol } 20 \% \text {. Dializador de } \\
\text { baja permeabilidad } \\
\text { Heparina Clexane } 40\end{array}$ & $\begin{array}{l}\text { Valoración del } \\
\text { paciente: } \\
\text { 1.Toma de } \\
\text { constantes } \\
\text { 2. Comprobación de } \\
\text { la permeabilidad de } \\
\text { la FAV } \\
\text { 3. Preparación para } \\
\text { el tratamiento } \\
\text { Seguimiento del Plan } \\
\text { de cuidados. } \\
\text { EPS ( } 1^{a} \text { sesión): die- } \\
\text { ta y hábitos } \\
\text { saludables. } \\
\text { Duración de trata- } \\
\text { miento (2a sesión): } \\
2 \text { h de HD. Sin pér- } \\
\text { didas } \\
\text { Perfusión continua } \\
\text { de Manitol } 20 \% \text {. } \\
\text { Dializador de baja } \\
\text { permeabilidad } \\
\text { Heparina Clexane } 40 \\
\text { (Al día siguiente de } \\
\text { la } 1^{a} \text { Sesión) }\end{array}$ & $\begin{array}{l}\text { Valoración del } \\
\text { paciente: } \\
\text { l.Toma de } \\
\text { constantes } \\
\text { 2. Comprobación } \\
\text { de la permeabili- } \\
\text { dad de la FAV } \\
\text { 3. Preparación pa- } \\
\text { ra el tratamiento } \\
\text { Seguimiento del } \\
\text { Plan de cuidados. } \\
\text { EPS ( }{ }^{\text {a }} \text { sesión): } \\
\text { Cuidados FAVI } \\
\text { Duración de trata- } \\
\text { miento ( } 3^{\mathrm{a}} \text { sesión): } \\
2 \mathrm{~h} 30 \text { minutos de } \\
\text { HD. Sin pérdidas. } \\
\text { Perfusión continua } \\
\text { de Manitol } 20 \% \text {. } \\
\text { Dializador de baja } \\
\text { permeabilidad } \\
\text { Heparina Clexa- } \\
\text { ne } 40 \\
\text { (Sesiones en días } \\
\text { alternos) }\end{array}$ & $\begin{array}{l}\text { Valoración del } \\
\text { paciente: } 1 . \text { Toma } \\
\text { de constantes } \\
\text { 2. Comprobación } \\
\text { de la permeabili- } \\
\text { dad de la FAV } \\
\text { 3. Preparación } \\
\text { para el } \\
\text { tratamiento } \\
\text { Seguimiento del } \\
\text { Plan de cuidados. } \\
\text { EPS (3a sesión): } \\
\text { Tratamiento } \\
\\
\text { Duración de } \\
\text { tratamiento } \\
\text { (4 sesión): } \\
3 \text { h de HD } \\
\text { Programar } \\
\text { ultrafiltración } \\
\text { según peso seco } \\
\text { Dializador FDX } \\
\text { 1,8 } \\
\text { Heparina Clexane }\end{array}$ & $\begin{array}{l}\text { Valoración del } \\
\text { paciente: } \\
\text { 1.Toma de constantes } \\
\text { 2. Comprobación de } \\
\text { la permeabilidad de } \\
\text { la FAV } \\
\text { 3. Preparación para el } \\
\text { tratamiento } \\
\text { Seguimiento del Plan } \\
\text { de cuidados: } \\
\text { repaso de } \\
\text { información aportada } \\
\text { Duración de trata- } \\
\text { miento (5a sesión): } \\
3 \text { h de HD. } \\
\text { Programar ultrafiltra- } \\
\text { ción según peso seco } \\
\text { Dializador FDX 1,8 } \\
\text { Heparina Clexane } 40 \\
\\
\text { Elaboración de ICC } \\
\text { Aportación al } \\
\text { paciente/cuidador } \\
\text { de encuesta de } \\
\text { satisfacción }\end{array}$ \\
\hline $\begin{array}{l}\text { AUXILIAR } \\
\text { ADMINIS- } \\
\text { TRATIV0 }\end{array}$ & $\begin{array}{l}\text { Gestión de } \\
\text { ambulancias }\end{array}$ & & & & & $\begin{array}{l}\text { Gestión de traslados. } \\
\text { Gestión de Alta y } \\
\text { derivación a Centro } \\
\text { periférico de diálisis } \\
\text { o a la unidad de } \\
\text { diálisis crónica }\end{array}$ \\
\hline $\begin{array}{l}\text { AUXILIAR } \\
\text { DE } \\
\text { ENFERMERÍA }\end{array}$ & $\begin{array}{l}\text { Tramitación y } \\
\text { programación de } \\
\text { inicio de paciente } \\
\text { en diálisis }\end{array}$ & $\begin{array}{l}\text { Entregará documento al } \\
\text { paciente/familia donde se } \\
\text { indique turno de diálisis }\end{array}$ & & & $\begin{array}{l}\text { Contactará con } \\
\text { centro periférico } \\
\text { o unidad de cróni- } \\
\text { cos el turno asig- } \\
\text { nado al paciente }\end{array}$ & \\
\hline $\begin{array}{l}\text { ESTUDIOS } \\
\text { COMPLE- } \\
\text { MENTARIOS }\end{array}$ & & $\begin{array}{l}\text { Marcadores (VIH, HB y } \\
\text { HC) ECG }\end{array}$ & & $\begin{array}{l}\text { Hemograma } \\
B Q \text { post }\end{array}$ & BQ post diálisis & \\
\hline MEDICACIÓN & & $\begin{array}{l}\text { Si hemoglobina por debajo } \\
\text { de } 7 \mathrm{~g} / \mathrm{dl} \text { o sintomatología } \\
\text { de anemia valorar trans- } \\
\text { fusión } \\
\text { EPO } 3000 \text { IV post diálisis } \\
\text { Venofer } 1 \text { vial IV/ semanal }\end{array}$ & $\begin{array}{l}\text { EP0 } 3000 \text { IV post } \\
\text { diálisis }\end{array}$ & $\begin{array}{l}\text { EP0 } 3000 \text { IV } \\
\text { post diálisis }\end{array}$ & $\begin{array}{l}\text { EP0 } 3000 \text { IV } \\
\text { post diálisis }\end{array}$ & $\begin{array}{l}\text { EP0 } 3000 \text { IV post } \\
\text { diálisis }\end{array}$ \\
\hline DIETA & & $\begin{array}{l}\text { No tomar nada durante la } \\
\text { diálisis }\end{array}$ & $\begin{array}{l}\text { No tomar nada } \\
\text { durante la diálisis }\end{array}$ & $\begin{array}{l}\text { No tomar nada } \\
\text { durante la diálisis }\end{array}$ & $\begin{array}{l}\text { No tomar nada } \\
\text { durante la diálisis }\end{array}$ & $\begin{array}{l}\text { No tomar nada } \\
\text { durante la diálisis }\end{array}$ \\
\hline
\end{tabular}

Matriz de la via clínica de inicio en hemodiálisis 


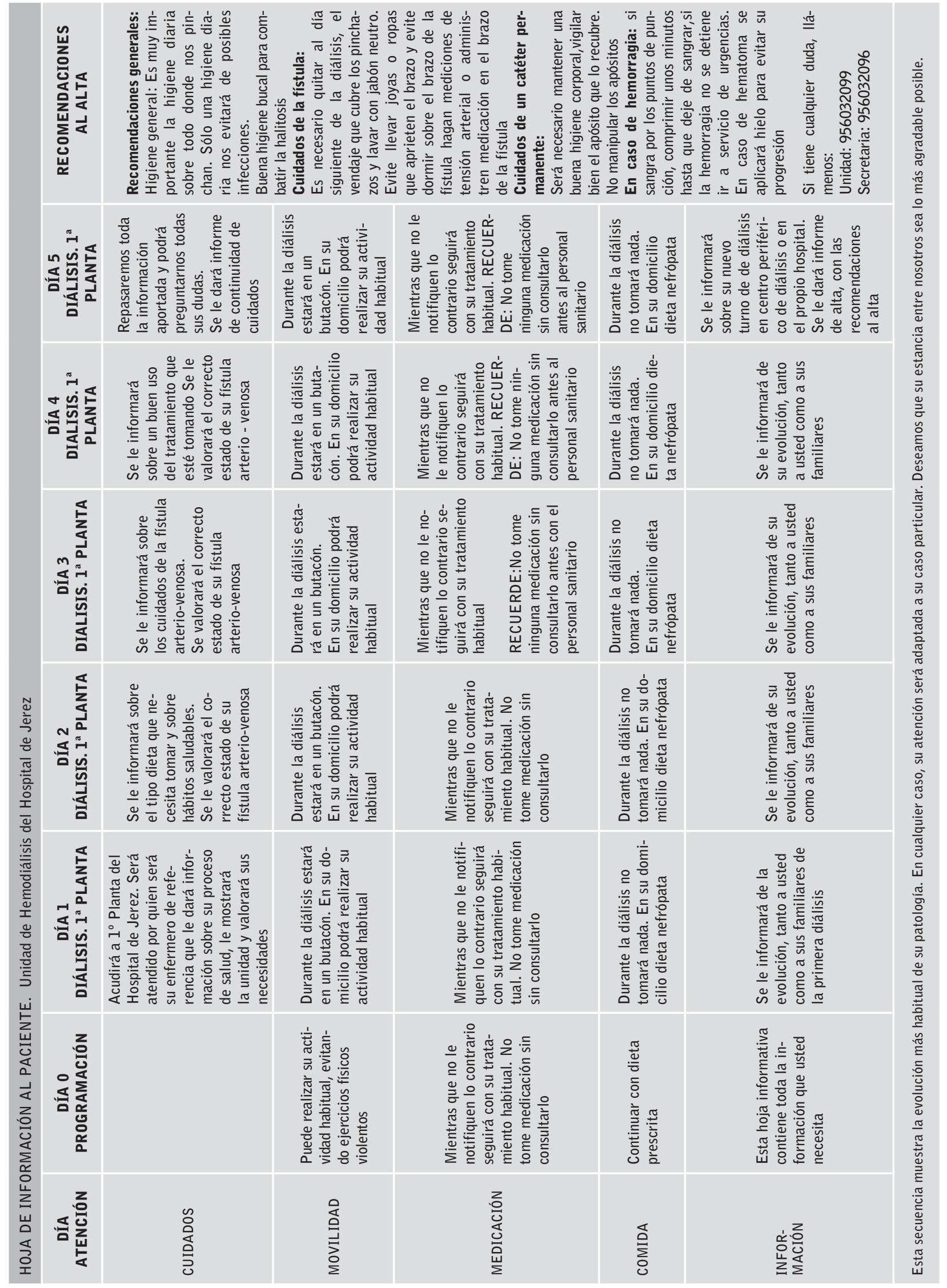


Recibido: 20 febrero 2012

Revisado: 10 Marzo 2012

Modificado: 12 Mayo 2012

Aceptado: 30 Mayo 2012

\section{Bibliografía}

1. Moisés Navarro Bermúdez. "Desarrollo e implementación de una vía clínica a los pacientes que inician la hemodiálisis de forma programada". Rev Soc Esp Enferm Nefrol 2011; 14 (1): 15/22

2. Proceso Asistencial Integrado: Tratamiento Sustitutivo de la Insuficiencia Renal Crónica: Diálisis y Trasplante Renal. Acceso [ 10 Enero 2012] disponible en: http://juntadeandalucia.es/salud/sites/ csalud/portal/index.jsp

3. Mira JJ, Aranaz J. La satisfacción del paciente como una medida del resultado de la atención sanitaria. Med Clin (Barc) 2000; 114 (Supl 3):26-33.

4. Contreras Abad, MD: Guía para pacientes con tratamiento de Hemodiálisis. Ed. Hospital Universitario Reina Sofía, S.A.S., Córdoba, 2001.
5. Sociedad Española de Nefrología. Normas de Actuación Clínica en Nefrología. Tratamiento Sustitutivo de la insuficiencia renal crónica: Tratamiento conservador. Madrid, 1999.

6. Gorriz JL, Sancho A, Pallardó LM. Significado pronóstico de la diálisis programada en pacientes que inician tratamiento sustitutivo renal. Un estudio multicéntrico español. Nefrología. Vol. XXII. $n^{\circ} 1.2002$.

7. Valdés $F$, Pérez Fontán $M$, Rodríguez-Carmona A, García Falcón T: Comienzo del tratamiento con diálisis. Nuevas tendencias. En: Aljama $P$, Arias $M$, Valderrábano: Insuficiencia renal progresiva. Editorial Grupo Entheos, Madrid, 2000.

8. Stratta R, Cushing K, Frisbie K, Miller S. Analysis of hospital charges after simultaneous pancreas-kidney transplantation in the era of managed care. Transplantation 1997; 64:287-92.

9. Holtzman J, Bjerke T, Kane R. The effects of clinical pathways for renal transplant on patient outcomes and length of stay. Medical Care 1998; 36:826-34. 